\title{
Squashed Single Feed Circularly Polarised Micro strip Antenna for Navigation Applications
}

\author{
Prasitha M, Yogeshwaran.A \\ (Communication Systems, Dhanalakshmi Srinivasan Engineering College/ Anna University, India) \\ (Communication Systems, Dhanalakshmi Srinivasan Engineering College/ Anna University, India)
}

\begin{abstract}
A squashed single-feed circularly-polarized (CP) wide beam microstrip antenna is projected for CNSS application. The antenna is intended by a double-layer arrangement, comprise a round space with two rectangular stubs the length of the width route and a sponging ring accurate more than it. To gather the uplink and downlink band of the scheme, a dual-band circularly polarized antenna should be encouraged and applied. In the meantime, due to the little number of CNSS satellites, the enormous gain of terminal antenna for small altitude is necessary. The third generation message scheme has been formally implement in our saleable. With the increasing difficulty of the scheme, it is straight away need to solve the multipath signal degradation and reduce the numeral, the cost and the site of antenna. For immediately gathering these supplies, the composite dual-polarized base location transmitter has been the prospect trend. The antenna is considered with a doublelayer arrangement, comprise a round patch with two rectangular stubs along the distance route and a parasitic ring right above it. The resonance frequency and the $C P$ character are mostly prohibited by the round patch and the rectangular stubs, correspondingly. The perpendicular HPBW could be widening by the parasitic ring. New results show that the deliberate vertical $H P B W$ is around 140 and the deliberate out-of-roundness for the horizontal radiation pattern is only $1.1 \mathrm{~dB}$. In addition, it could uphold good inconspicuous character.
\end{abstract}

Keywords: circular patch, $H P B W$, parasitic ringing effect, Polarisation, Out of roundness.

\section{Antenna and Wave Propagation}

\section{Introduction:}

Radio signal are a form of electromagnetic wave, and as they be the technique in which means of communication signal travel, they have a main bearing on RF antenna themselves and RF transmitter design. Electromagnetic waves are the same category of emission as light, ultra-violet and infra red rays, conflicting from them in their wavelength and frequency. Electromagnetic waves have both electric and magnetic components that are inseparable. The planes of these fields are at right angles to one another and to the direction of motion of the wave.

\section{Faraday Prinicple:}

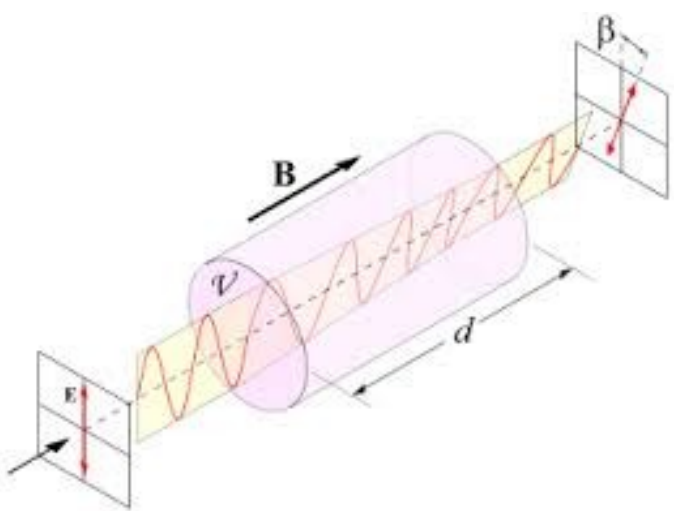

Fig 1: faraday principle

Faraday rotary motion in the ionosphere is cause by the communication between a propagate Electromagnetic gesticulate and the Earth's attractive field. In universal, the point of reference angle (or tilt angle, T) of a linearly polarized microwave change when the wave pass. During the ionosphere, up to now, only linearly polarized microwaves (both vertical and horizontal polarization) have been subjugated in Space bear synthetic aperture Radar (SAR) systems. Thus, the present linearly Polarized SAR (LP-SAR) systems are predictably exaggerated by Faraday rotation in both Downward (from a satellite to the Earth's surface) and 
upward (reflected from the Earth's surface) broadcast. The method of double Faraday alteration is wanted to right the deliberate data. As T turning round is larger for longer wavelengths, in do the L-band frequency (1.27 $\mathrm{GHz}$ ) is most considerably affected; in the nastiest case, a rotation Of approximately 40 Is expected for microwaves with linear polarization.

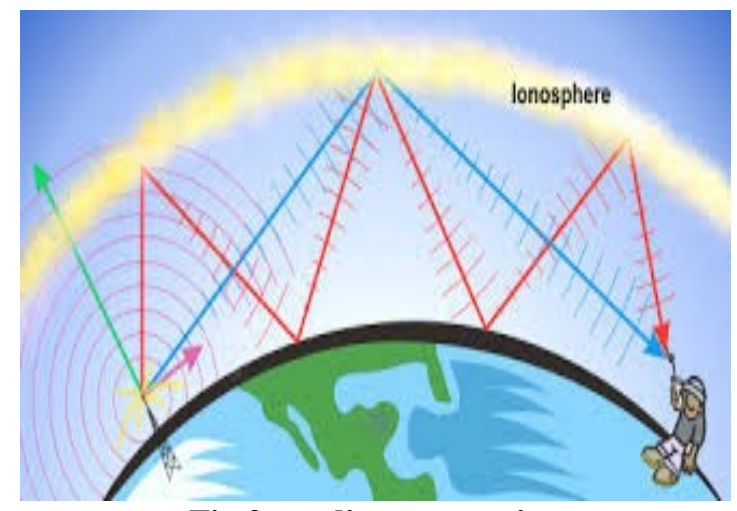

Fig 2: radio propagation

\section{Sattelite:}

Satellites are extensively used these days for all from direction-finding, in the container of GPS, satellite television dissemination, communication, mobile phone skill, Internet broadband weather monitoring and much more.

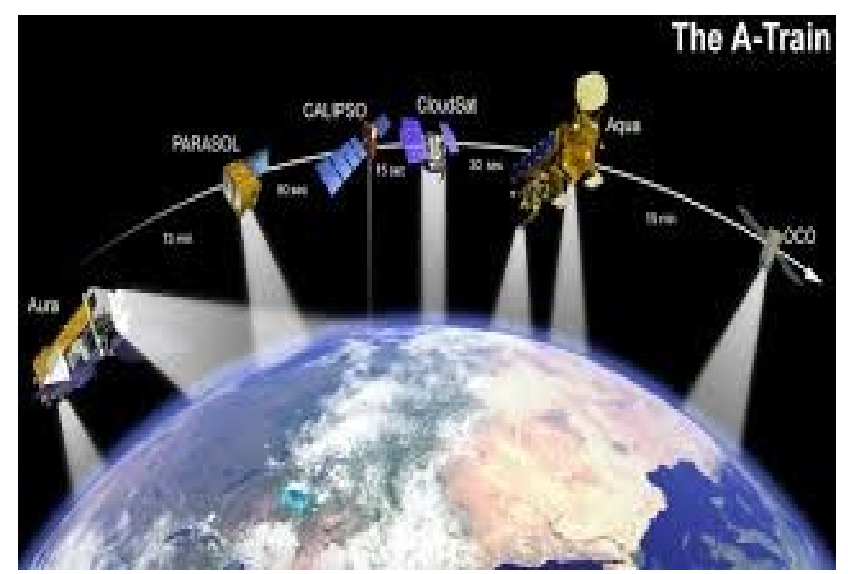

Fig 3: Satellite system

Satellites usually use frequencies that be in excess of $500 \mathrm{MHz}$ where the signals are not excessively exaggerated by the ionosphere or troposphere. However some belongings can be notice and are important, particularly when preparation, install or location up a satellite system. When signals pass through from the earth up to the satellite they pass through four main regions. These are the troposphere, above that is region that is often term interior free space which is greater than the troposphere and less the ionosphere. The next region is the ionosphere, and finally there is the outer free space. The troposphere and ionosphere have refractive indices that different from unity. The troposphere is better than agreement and the ionosphere is less than agreement and as product refraction and absorption occur. The inner free space region also has small result.

\section{Related Work:}

In [1] xidong wu, George v. eleftheriades t. Emilie van deventer-perkins presents Design and characterization of single- and multiple-beam mm-wave circularly polarized substrate lens antennas for wireless communications. This aim incidence has been chosen partially due to the variety allotment for at present planned mm-wave wireless systems and partially due to offered laboratory tools. Circular split and high directivity are attractive features, as they preserve a CP single-beam antenna is earliest introduce based on a low-cost with no trouble machined rexolite substrate lens. For the single-beam plan, an ellipsoidal lens with a cylindrical conservatory slab is utilize, which leads to diffraction limited pattern on axis. The chosen nourish is an aperture-coupled scrap antenna, which minimize back emission arise from the low down permittivity of rexolite. 
In [2] Pengcheng Li, Jianxin Liang, Xiaodong Chen presents Study of Printed Elliptical/Circular Slot Antennas for Ultra wideband Applications. A U-shaped change stub is introduce to improve the combination among the slot and the feed line so as to thicken the in service bandwidth of the antenna. In addition, an added bandwidth improvement can be achieve by narrowing the feed line. Good concurrence is obtain between the replication and testing. Both of them cover exposed that the proposed antenna can show UWB typical with nearly Omni directional emission patterns over the entire bandwidth. The ultra wide bandwidth of the slit antenna consequences from the overlap of the multiple resonances introduce by the mixture of the oval slot and the feed line with U-shaped change stub.

In [3] C. Rizki Akbar, J. Tetuko s. S. And h. Kuze presents A novel circularly polarized synthetic aperture radar (CP-SAR) system onboard a space borne platform. The use of circular polarization can provide a solution to the Faraday rotation problem in many microwave systems; it has not previously been applied to SAR systems. In this paper, we describe the novel concept of a Space borne SAR system based on circular polarization, and show that a smaller antenna size, lower transmitted peak power and high precision signal reconstruction can be achieved by the proposed circularly polarized (CP)-SAR system.

In [4] Mark J. Vaughan, Katarina Y. Hur, and Richard C. Compton presents Improvement of Micro strip Patch Antenna Radiation Patterns. The metal layers on also side of the dielectric describe a similar plate waveguide which is open-circuited at every end. The majority of the authority in the gesticulate keyed up in the guide is reflect back at the two ends; some is transmit through the dielectric beyond the metal in outside wave mode, and the rest is radiate from the fringing emotional fields on the limits of the patch. Since of the large reflection coefficients, confine the power between the metal layers, and the power vanished to surface waves, this antenna is careful very ineffective. Simple transmit line model for the piece antenna sight it as a halfwavelength extended micro strip line with opening antennas at both trimmings.

In [5] K. Chen1, H. Wang1, Y. Huang2 and J. Wang presents A Compact Dual-Band Assembled Printed Quadrafilar Helix Antenna for CNSS Application. Achieve the axial emission model at B1 band; in addition, it produces the shaped-conical emission outline at $\mathrm{S}$ band that is promote for getting the course-plotting signal from low altitude angle. Simulations confirm that 10dB impedance bandwidth of the projected novel dense CNSS dual-band PQHAs are $15.4 \%$ at B1 group and $14.2 \%$ at S group. A very good-looking aspirant for these application is the resonant Quadrafilar helical antenna and more lately the conservative in print Quadrafilar helical antenna owing to their performance in terms of circular division, good axial ratio (AR), light weight, high dimensional constancy, ease of manufacture and little cost.

\section{Compact Dual Band Circular Patch Antenna}

\subsection{Quadrifilar Helix Antenna}

- Quadrafilar helix antenna has good circular polarization character. Its heart-shaped emission pattern, which leads to wide perpendicular HPBW and elevated gain for low height angle, could meet the insist of satellite communication. However, the profile of Quadrafilar helix antenna is also high to be coplanar and included with message systems.

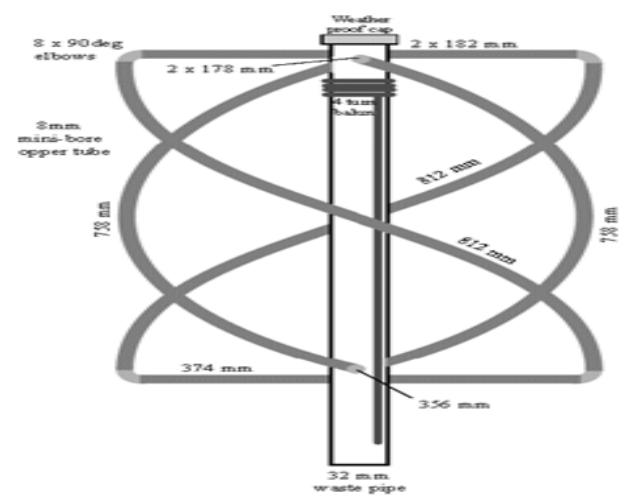

Fig 4: Quadriflar Helix Antenna

\subsection{Microstrip Antenna}

- Micro strip antenna has many compensation such as near to the ground outline, light weight, easy manufacture and conformability to rising hosts. 
- In the meantime it might be easy to make good circular division individuality and since of this it has been highly valued. Regularly, the vertical HPBW of micro strip antenna is concerning 70-110.

- On the affirmation of curved divergence character, how to enlarge the plumb HPBW and movement the get for low augment outlook are especially hard research topics at current.

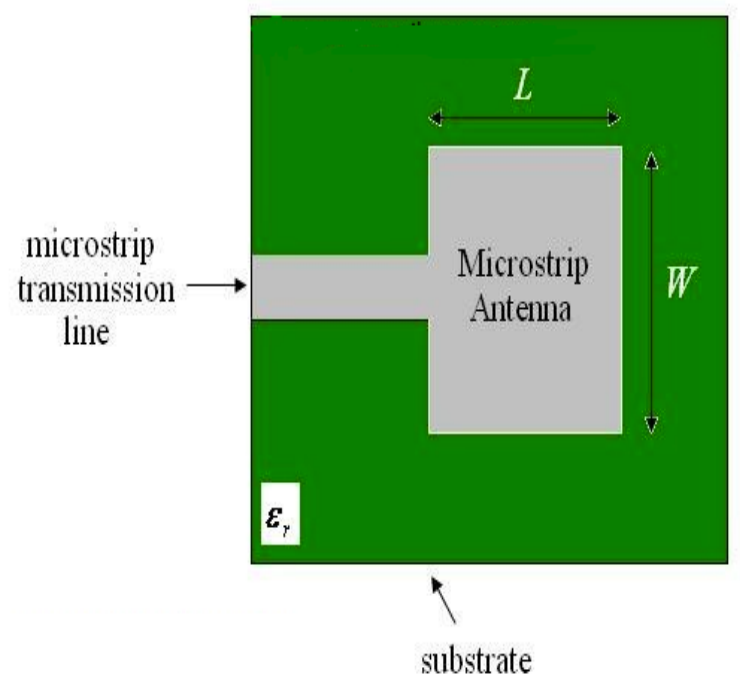

Fig 5: Microstrip antenna

\subsection{Circular Polarization}

In electrodynamics, round polarization of an electromagnetic wave is a polarization in that the electric meadow of the transitory gesticulates does not alter power but only change way in a rotating manner. In electrodynamics the power and route of an electric field is distinct by an electric field vector. In the case of a circularly polarized wave, as seen in the complementary liveliness, the tilt of the electric turf vector, at a set point in gap, describe a circle as instant progresses. If the signal is unmoving in occasion, the electric field vector of the wave describes a helix the length of the way of spread.

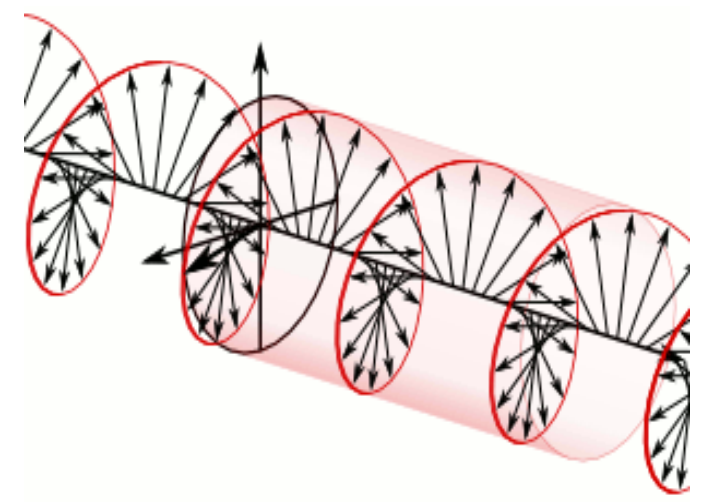

Fig 6: Circular polarization

\subsection{Antenna Design:}

The higher one is single-side metal arrangement and the scrounging emission circle is on the peak. The radii use of the external and inner ring is $\mathrm{R} 1$ and $\mathrm{R} 2$, in that order. The lesser one is a bilateral metal construction, of which the base is the floor and the higher is the key emission plot of the antenna. The major radiation patch comprises a circle with radius R3 and two rectangular stubs along the width direction. Each stub have a width of $\mathrm{W}$ and is at a distance of $\mathrm{L} 1$ commencing the substrate center. The width of the gap connecting these two substrates is $\mathrm{h}$, which is normally chosen less than $\frac{\lambda}{8}$ in order to maintain the unremarkable uniqueness of micro strip antennas. 


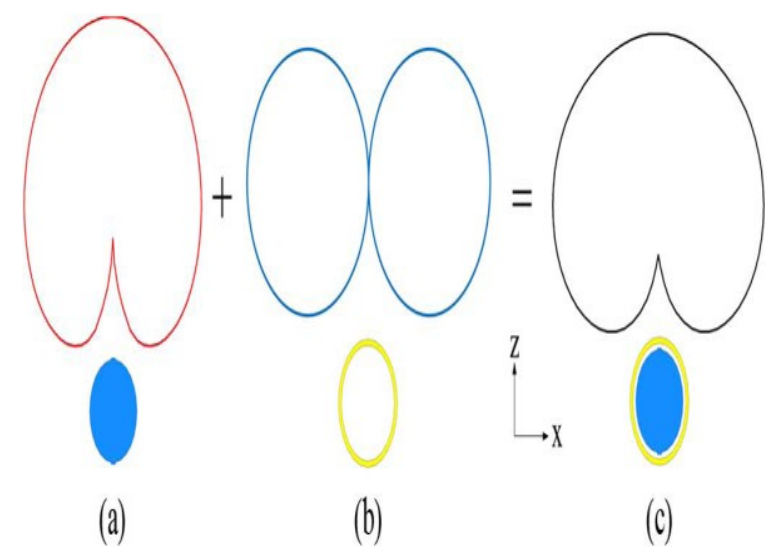

Fig 7: Antenna pattern

The main emission patch of the future antenna is fed by means of 50 SMA. The nourish point is situated on 45 degree slanting of the width along the two rectangular stubs. The difference between the supply point and the core of the main emission patch is L2. The exterior current animated by the feed point could be determined into two parts, one down and the other upright to the rectangular stubs. With the two rectangular stub inserted, the previous path is lingering while the latter is only just artificial. Therefore, there is length dissimilarity connecting the two instructions. In other words, the surface current might be tearing into two neardegenerate mode with equivalent amplitude and 90 phase move for CP individuality. By adjust the stub span and the distance between the supply point and the patch core, good circularly-polarized individuality could be achieved. The parasitic ring piece is fed by air combination.

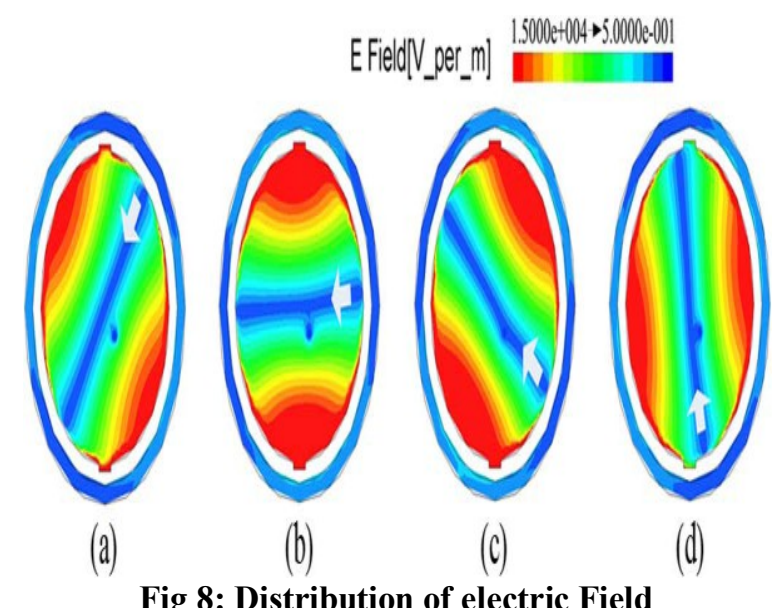

Fig 8: Distribution of electric Field

\subsection{Effects Of The Parasitic Ring}

It is experiential that, before and after load the scrounging ring, the LHCP gain for 90 height angle are in that order $3.52 \mathrm{~dB}$ and $6.58 \mathrm{~dB}$ and the plumb HPBW are in that order 90 and 131 . The average gains for 5 elevation angle are respectively $2.56 \mathrm{~dB}$ and $1.45 \mathrm{~dB}$. Therefore the emission pattern similar to tire form generates by the scrounging ring, could reduce the gain for 90 height angle and improve the increase for low elevation angle. In other words, the vertical HPBW of the future antenna is clearly wider with the placing of the scrounging ring.

\subsection{Effects Of Outer Radius Of The Parasitic Ring}

The simulated results of total gain for the projected antenna with dissimilar external radius of the parasitic ring. With R1 amplified from $20 \mathrm{~mm}$ to $21 \mathrm{~mm}$, the gain for 90 height angles is better while the one for 5 elevation position is decrease. So the upright HPBW of the future antenna could be widening by falling the outer radius.

\section{Simulation Results:}

According to the results of the numerical analysis, the optimized parameters of the proposed antenna dimensions are as follows: $\mathrm{L} 1=17 \mathrm{~mm}, \mathrm{~L} 2=3.8 \mathrm{~mm}, \mathrm{R} 1=20.5 \mathrm{~mm}, \mathrm{R} 2=18.5 \mathrm{~mm}, \mathrm{R} 3=16.4 \mathrm{~mm}, \mathrm{w}=3 \mathrm{~mm}, \mathrm{~h}=10 \mathrm{~mm}$. The overall size of the proposed antenna is and the photo of the fabricated antenna 


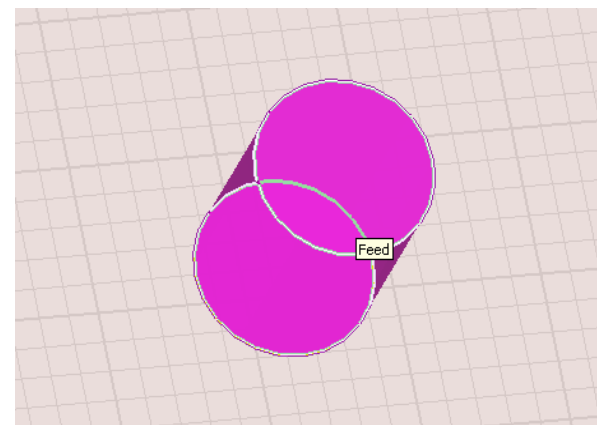

Fig 9: Designed antenna

The structural parameters of the parasitic ring are key factors for determining the vertical HPBW. Besides, it could maintain good omnidirectional characteristics for the horizontal radiation pattern. This structure is simple and easy to manufacture.

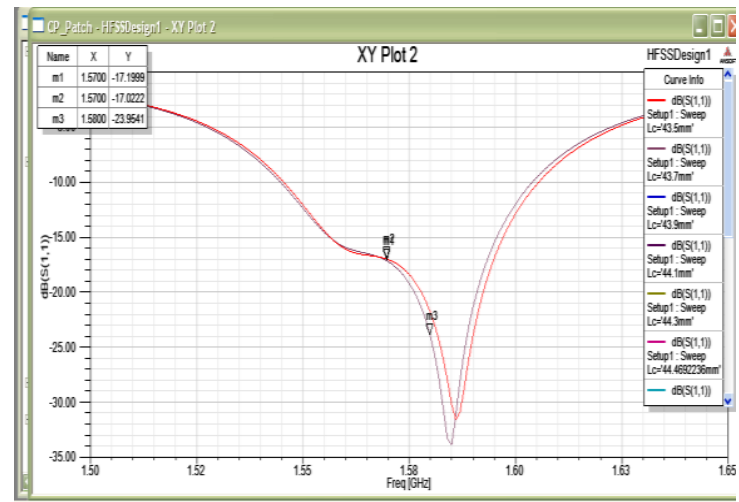

Fig 10: Measured S11 of antenna

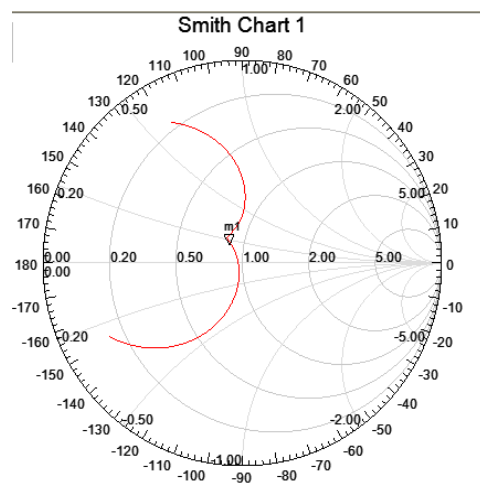

Fig 11: Smith Chart

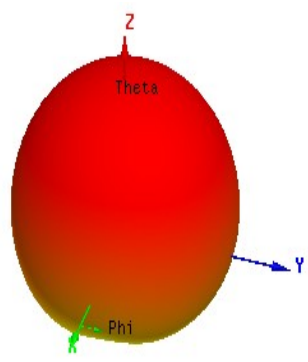

Fig 12: 3D Polar Plot 


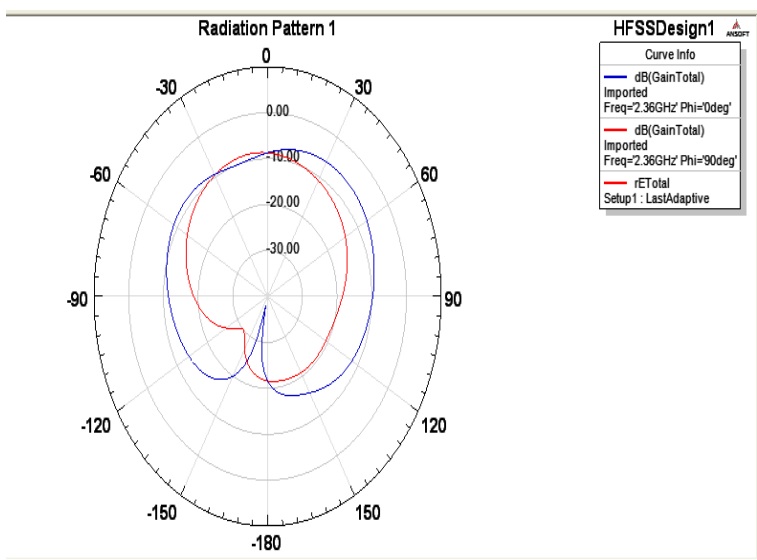

Fig 13: Radiation Pattern

\section{Conclusion:}

A novel dense circularly-polarized antenna with parody slot for CNSS dual-band application is here. The aerial agreement is simple and easy to process. In adding; it can mainly reduce the size of the antenna and meet the some especially smallness supplies. These planned antennas are very simple, cost successful and high competence for the application in GHz incidence ranges. The optimum proposes is old to achieve the compact dimensions and high radiation efficiency. A new configuration of micro strip series-fed decrease array is intended in order to progress the presentation of micro strip aerial array. This technique is base on a parasitic circle right above the micro strip antenna. A prototype antenna based on the proposed method was designed, at $2.492 \mathrm{GHz}$ for CNSS application, fabricated and tested. The measurements showed it could effectively widen the vertical HPBW and improve the gain for low elevation angle. The structural parameters of the parasitic ring are key factor for formative the vertical HPBW. Besides, it could uphold good unidirectional individuality for the flat radiation outline. This structure is simple and easy to manufacture.

\section{References}

[1]. Inside GNSS, Compass Beidou Inside GNSS [Online]. Available: http://www.insidegnss.com/compass 2013

[2]. J. Z. Liu, Y. H. Huang, and M. Lei, "Dual-Band Wide-Beam Circularly- Polarized Antenna With Good Gain of Low Elevation Angle," Chinese patent, CN2865037Y.

[3]. C. Kilgus, "Shaped-conical radiation pattern performance of the backfire Quadrafilar helix," IEEE Trans. Antennas Propag., vol. 23, no. 3, pp. 392-397, 1975.

[4]. O. P. Leisten, "Miniaturized dielectrically-loaded Quadrafilar antenna for global positioning system (GPS)," Electron. Lett., vol. 37, no. 22, pp. 1321-1322, 2001.

[5]. Q. X. Chu, W. Lin, W. X. Lin, and Z. K. Pan, “Assembled dual-band broadband Quadrafilar helix antennas with compact power divider networks for CNSS application,” IEEE Trans. Antennas Propag., vol. 61, no. 2, pp. 516-523, 2013.

[6]. K. P. Yang and K. L. Wong, "Dual-band circularly-polarized square micro strip antenna," IEEE Trans. Antennas Propag., vol. 49, no. 3, pp. 377-382, 2001.

[7]. Q. X. Chu and W. Liao, "Dual-band circularly polarized micro strip antenna with small frequency ratio," Progr. Electromagnet. Res. Lett., vol. 15, pp. 145-152, 2010.

[8]. Y. Sung, "Dual-band circularly polarized pentagonal slot antenna," IEEE Antennas Wireless Propag. Lett., vol. 10, pp. 259-261, 2011.

[9]. S. Foo and B. Vassilakis, "Dielectric fortification for wide-beam width patch arrays," presented at the Antennas and Propagation Society Int. Symp., IL, USA, 2008.

[10]. H. D. He, "A novel wide beam circular polarization antenna-micro strip- dielectric antenna," presented at the Int. Conf. on Microwave and Millimeter Wave Technology, Beijing, China, 2002. 